\title{
Development of an Ex Vivo, Beating Heart Model for CT Myocardial Perfusion
}

\author{
Gert Jan Pelgrim, ${ }^{1}$ Marco Das, ${ }^{2}$ Ulrike Haberland, ${ }^{3}$ Cees Slump, ${ }^{4}$ \\ Astri Handayani, ${ }^{1}$ Sjoerd van Tuijl, ${ }^{5}$ Marco Stijnen, ${ }^{5}$ Ernst Klotz, ${ }^{3}$ Matthijs Oudkerk, ${ }^{6}$ \\ Joachim E. Wildberger, ${ }^{2}$ and Rozemarijn Vliegenthart ${ }^{1}$ \\ ${ }^{1}$ University of Groningen, University Medical Center Groningen, Center for Medical Imaging-North East Netherlands, \\ Department of Radiology, Hanzeplein 1, 9713 GZ Groningen, Netherlands \\ ${ }^{2}$ Department of Radiology and Cardiovascular Research Institute Maastricht (CARIM), Maastricht University Medical Center, \\ Postbus 5800, 6202 AZ Maastricht, Netherlands \\ ${ }^{3}$ Siemens AG Healthcare, Forchheim, Germany \\ ${ }^{4}$ University of Twente, Drienerlolaan 5, 7522 NB Enschede, Netherlands \\ ${ }^{5}$ LifeTec Group BV, Den Dolech 2, 5612 AZ Eindhoven, Netherlands \\ ${ }^{6}$ University of Groningen, University Medical Center Groningen, Center for Medical Imaging-North East Netherlands, \\ Hanzeplein 1, 9713 GZ Groningen, Netherlands
}

Correspondence should be addressed to Rozemarijn Vliegenthart; r.vliegenthart@umcg.nl

Received 11 September 2014; Accepted 23 December 2014

Academic Editor: Marco Francone

Copyright ( 2015 Gert Jan Pelgrim et al. This is an open access article distributed under the Creative Commons Attribution License, which permits unrestricted use, distribution, and reproduction in any medium, provided the original work is properly cited.

Objective. To test the feasibility of a CT-compatible, ex vivo, perfused porcine heart model for myocardial perfusion CT imaging. Methods. One porcine heart was perfused according to Langendorff. Dynamic perfusion scanning was performed with a secondgeneration dual source CT scanner. Circulatory parameters like blood flow, aortic pressure, and heart rate were monitored throughout the experiment. Stenosis was induced in the circumflex artery, controlled by a fractional flow reserve (FFR) pressure wire. CT-derived myocardial perfusion parameters were analysed at FFR of 1 to 0.10/0.0. Results. CT images did not show major artefacts due to interference of the model setup. The pacemaker-induced heart rhythm was generally stable at 70 beats per minute. During most of the experiment, blood flow was $0.9-1.0 \mathrm{~L} / \mathrm{min}$, and arterial pressure varied between 80 and $95 \mathrm{~mm} / \mathrm{Hg}$. Blood flow decreased and arterial pressure increased by approximately $10 \%$ after inducing a stenosis with FFR $\leq 0.50$. Dynamic perfusion scanning was possible across the range of stenosis grades. Perfusion parameters of circumflex-perfused myocardial segments were affected at increasing stenosis grades. Conclusion. An adapted Langendorff porcine heart model is feasible in a CT environment. This model provides control over physiological parameters and may allow in-depth validation of quantitative CT perfusion techniques.

\section{Introduction}

Computed tomography (CT) has become the premier noninvasive imaging modality for the noninvasive evaluation of the coronary arteries. For the functional assessment of coronary artery disease (CAD), the sole diagnosis of coronary luminal narrowing is often limited, especially in case of 30-70 percent (intermediate) grade stenosis [1]. Usually, additional testing on the impact of stenosis on myocardial perfusion is needed. To date, CT is not commonly used in daily clinical practice worldwide, except for several leading clinics in CT imaging. However, recent evidence suggests that state-of-theart CT scanners allow evaluation of myocardial blood supply, on top of the interrogation of coronary morphology [2] This includes quantification of myocardial perfusion using dynamic perfusion techniques in second-generation dual source CT (DSCT) scanning [3, 4]. Measurement of absolute myocardial perfusion can enhance the diagnostic accuracy for hemodynamically significant $\mathrm{CAD}$, compared to visual analysis of perfusion maps [4-6]. At present, only positron emission tomography (PET) imaging is capable of true perfusion quantification [7]. Morton et al. recently demonstrated 

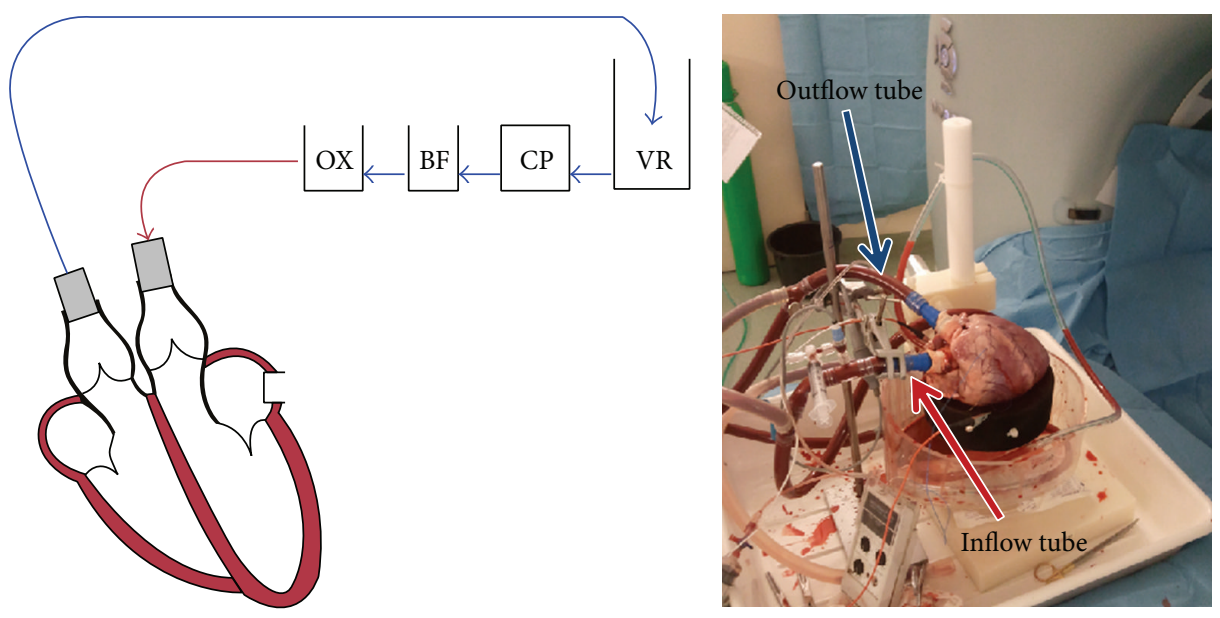

FIGURE 1: From the venous reservoir (VR), the blood first passed the cardiac pump CP. Then, the blood was pumped through the blood filter (BF) into the oxygenator (OX) and, from there, through the aorta into the coronary arteries. The setup was placed on the scanner table; all parts which could possibly interfere with the signal were placed outside the field of view.

potential for CMR to derive semiquantitative parameters of perfusion [8].

Before quantitative CT perfusion imaging can be implemented in clinical practice, systematic validation is mandatory. Therefore, an ex vivo model of a perfused, isolated heart of a slaughterhouse pig was used for this systematic analysis.

The aim of this study was to develop an explanted, perfused porcine heart model in a CT environment. We hypothesized that this model allows for standardized validation of CT perfusion parameters.

\section{Materials and Methods}

2.1. Heart Acquisition and Preparation. In this experiment, one ex vivo porcine heart model was used. The current study was designed to purely show the feasibility of the model. The heart was obtained from a pig, slaughtered for human consumption. Protocols at the slaughterhouse and laboratory were in accordance with EC regulations 1069/2009 regarding the use of slaughterhouse animal material for diagnosis and research, supervised by the Dutch Government (Dutch Ministry of Agriculture, Nature and Food Quality) and approved by the associated legal authorities of animal welfare (Food and Consumer Product Safety Authority).

A Dutch Landrace hybrid pig of approximately $110 \mathrm{~kg}$ live weight was used. After exsanguination, the thorax was opened by a parasternal incision. The pulmonary artery was cut just before the bifurcation. The aorta was cut before the supra-aortic vessels. The heart was immediately cooled topologically in ice slurry. To arrest the heart, the aorta was cannulated to administer $2 \mathrm{~L}$ of cold cardioplegic solution $\left(4^{\circ} \mathrm{C}\right.$ Custodiol histidine-tryptophan-ketoglutarate (HTK), Essential Pharmaceuticals, Pennsylvania, USA) to the coronary arteries at a pressure of $60 \mathrm{mmHg}$. The ischemic time at body temperature did not exceed 5 minutes. From subsequently slaughtered pigs, $20 \mathrm{~L}$ of fresh blood was collected for reperfusion. The heart and blood (heparinized with 5000 IU/L) were stored cold during transportation. Preparations of the heart were executed in the laboratory under cold and cardioplegic conditions until resuscitation. First, the pericardial sack was discarded. The azygos vein and the inferior and superior caval vein were ligated. A cannula was inserted into the aorta and fixed approximately $40 \mathrm{~mm}$ distal to the valve annulus. The pulmonary veins were cut at the left atrium and ligated. A perforated drain cannula was inserted into the left atrium and ventricle allowing sufficient venting after resuscitation. Similarly, a cannula was inserted into the pulmonary artery. Around the proximal circumflex $(\mathrm{Cx})$ coronary artery, an inflatable cuff was placed, providing the possibility of mimicking stenosis under controlled circumstances. By measuring pressure distal to the stenosis and calculating the ratio between this pressure and the aortic pressure, the fractional flow reserve (FFR) was determined, a measure for the hemodynamic severity of the stenosis. In clinical practice, FFR measurements are performed under maximal hyperaemic conditions, using adenosine or dipyridamole. The heart in this experiment was already in maximal hyperaemic state, because of its removal from the pig body [9]. The Cx artery was chosen because it is easily accessible (in contrast to the right coronary artery) and perfuses an identifiable, but not too large, part of the left ventricle (in contrast to the left anterior descending artery). During the experiment, FFR measurements were used to verify the cuff-induced stenosis grade. CT perfusion scanning was first performed without stenosis, followed by scanning at FFR values of $0.7,0.5,0.3$, and 0.1/0.0. Scanning was repeated three times at each FFR. A deviation of 0.05 from the goal FFR was considered acceptable. To prevent the contrast agent from building up in the circulating blood pool and thus causing differential baseline enhancement, the blood pool was refreshed with new blood after each stenosis grade situation.

2.2. Heart Perfusion. After preparation, the aorta and pulmonary artery were connected to the circulation loop using the cannulas (Figure 1). A bed of flexible cloth provided epicardial suspension. The heart was aligned in the scanner 
in supine position. A modified Langendorff perfusion model was used, with an artificial heart-lung loop. In 1895, Langendorff et al. proposed a model of retrograde perfusion of mammalian hearts in which a Krebs-Henseleit solution circulated via the aorta $[10,11]$. This Langendorff model was refined by circulation of whole blood [12]. Whole blood was pumped by a centrifugal pump (BioMedicus, Medtronic, Minneapolis, MN, USA) from a venous reservoir into the aorta towards the aortic sinus and the coronary arteries. The flow in the coronaries is pulsatile and pressure dependent. A pressure is present at the aortic root, causing the flow of blood into the coronaries. When the myocardium contracts during systole, the coronary vasculature is compressed and therefore the vascular resistance increases, resulting in reduced coronary flow. During diastole the myocardium relaxes, which opens the vascular bed and thereby lowers vascular resistance with higher coronary flow as a result. In a normally functioning heart, the aortic blood pressure pulse varies in pressure between 120 and $80 \mathrm{mmHg}$, resulting in a pressure pulse of $40 \mathrm{mmHg}$. However, in the Langendorff experiments the pressure pulse is only in the order of magnitude of $10 \mathrm{mmHg}$.

The coronary venous blood returned to the venous reservoir via the coronary sinus, right atrium, right ventricle, and pulmonary artery. The reperfusion medium circulated through a filter (AFFINITY Arterial $38 \mu \mathrm{m}$ blood filter; Medtronic, Minneapolis, Minnesota, USA) and an oxygenator-heat exchanger (AFFINITY NT Oxygenator; Medtronic, Minneapolis, Minnesota, USA). The blood was oxygenated with $20 \% \mathrm{O}_{2}, 75 \% \mathrm{~N}_{2}$, and $5 \% \mathrm{CO}_{2}$ carbogen gas. Blood glucose level was maintained between 5 and $7 \mathrm{mmol} / \mathrm{L}$ by addition of glucose-insulin mixture. The temperature of the circulating blood was $38^{\circ} \mathrm{C}$. The blood flow through the coronary arteries was approximately $1.5 \mathrm{~mL} / \mathrm{g}$ per minute and controlled with the centrifugal pump. The mean coronary flow was measured with an ultrasound flow probe (LifeTec Group, Eindhoven, Netherlands) at the pump, and the pressure was measured at the aortic root with a pressure sensor (P10EZ-1; Becton Dickinson Medical, Franklin Lakes, New Jersey, USA).

When reperfusion of the coronary system was started, total ischemic time was approximately 4 hours. After coronary perfusion was reinstated, the heart showed spontaneous contractions while warming up by the circulating whole blood and gradually regained sinus rhythm. Some defibrillations were performed, of 10-30 Joules, to restore sinus rhythm. The heart was kept in this condition for at least 15 minutes, to allow stabilisation at the membranal level. If the heart rhythm did not stabilize, or if rhythm became irregular during the experiment, a Medtronic external pacemaker model 5375 (Medtronic, Minneapolis, Minnesota, USA) was used to induce a stable heart rate at approximately 70 beats per minute (see movie in Supplementary Material available online at http://dx.doi.org/10.1155/2015/412716). After obtaining a stable heart rate, the imaging protocols were started. ECG clips were placed on the "wet" flexible cloth and connected to the scanner ECG leads to feed signal to the scanner and allow ECG-synchronized image acquisition. During the entire experiment, the heart rate, pacing status (heart paced yes or no), aortic pressure, and blood flow were monitored.
This enabled analysis of model stability and influence of luminal narrowing on the model.

2.3. CT Imaging Protocol. CT acquisitions were performed on a second-generation DSCT scanner (SOMATOM Definition Flash, Siemens Healthcare, Forchheim, Germany). Scans were acquired in caudocranial direction. At each stenosis grade, the protocol consisted of a noncontrast enhanced CT scan, three dynamic CT perfusion (CTP) acquisitions, and one coronary CT angiography (CTA) scan. First, scout images were obtained to determine the area of interest and a noncontrast enhanced CT scan was performed to determine baseline enhancement of the heart and blood pool. The unenhanced scan was made at tube voltage of $100 \mathrm{kV}$ and tube current of $100 \mathrm{mAs}$. Next, perfusion series were performed at 5 -minute intervals, to allow mixing of contrast agent in the blood pool and thus minimize the influence of contrast buildup. Dynamic perfusion image acquisitions were performed during end-systole (300 ms delay after the R-wave) in shuttle mode, with ECG-triggering. Given a detector width of $38.4 \mathrm{~mm}$ and an overlap of $10 \%$, the anatomical coverage was $7.3 \mathrm{~cm}$ [13]. The scan area was determined based on the scout scan. The entire left ventricle of the heart was selected as image field of view (FOV), from the aortic root to the apex. The heart was placed parallel to the $z$-axis of the table. Matrix size was $512 \times 512$. Due to a heart frequency of $>70$ per minute, images were acquired every second heartbeat. Image acquisition parameters were $2 \times 2 \times 64$ detector rows, $3.0 \mathrm{~mm}$ collimation, $2 \times 100 \mathrm{kV}$ tube voltage, and $350 \mathrm{mAs}$ per rotation with a rotation time of $285 \mathrm{~ms}$. The CM concentration was $300 \mathrm{mg}$ iodine $/ \mathrm{mL}$ (Ultravist 300, Bayer, Berlin, Germany), which was diluted to a mixture of 40 percent contrast and 60 percent saline for the injections. CM was injected into the blood stream $200 \mathrm{~cm}$ from the coronary arteries, allowing mixing of the perfusate and the $\mathrm{CM}$ but preserving the bolus shape of the injection. A CM volume of $15 \mathrm{~mL}$, with a contrast to saline ratio of $40 / 60$, was used at an injection rate of $3 \mathrm{~mL} / \mathrm{sec}$. Imaging was started 5 seconds prior to start of CM injection, with a total scan time of 35 seconds. After the perfusion series, coronary CTA scanning was performed. First, scan timing was determined by administering additional $10 \mathrm{~mL}$ of diluted (40/60 contrast/saline ratio) iodine contrast. Image acquisition was initiated 3 seconds after peak enhancement in the aortic root. Then, CTA was performed with another $15 \mathrm{~mL}$ of diluted contrast at an injection rate of $3 \mathrm{~mL} / \mathrm{sec}$. Coronary CTA data were acquired with retrospective ECG-gating, to analyse best-systolic and best-diastolic phase reconstructions. Scan parameters were $2 \times 2 \times 64$ detectors rows, $0.6 \mathrm{~mm}$ detector collimation, $2 \times 100 \mathrm{kV}, 350 \mathrm{mAs}$ per rotation, and $285 \mathrm{~ms}$ gantry rotation time. CTA data were acquired to analyse the stenosis severity related to the FFR measurements.

2.4. Image Reconstruction. Coronary CTA datasets were reconstructed with $0.75 \mathrm{~mm}$ slice thickness, $0.3 \mathrm{~mm}$ increment, and I26F (iterative) reconstruction kernel to reduce image noise. Coronary evaluation was performed in Syngo.via, based on curved multiplanar reformat series. The degree of luminal narrowing of the different stenosis grades 
TABLE 1: Arterial blood flow (L/min) and blood pressure $(\mathrm{mm} / \mathrm{Hg})$ in 5 different stenosis grades.

\begin{tabular}{lccccc}
\hline & Normal perfusion & FFR 0.70 & FFR 0.50 & FFR 0.30 & Total occlusion \\
\hline Arterial blood flow (SD) & $\begin{array}{c}1.0 \mathrm{~L} / \mathrm{min} \\
\text { (constant) }\end{array}$ & $\begin{array}{c}1.0 \mathrm{~L} / \mathrm{min} \\
\text { (constant) }\end{array}$ & $\begin{array}{c}1.0 \mathrm{~L} / \mathrm{min} \\
\text { (constant) }\end{array}$ & $\begin{array}{c}0.93 \mathrm{~L} / \mathrm{min} \\
(\mathrm{SD} \mathrm{0.05)}\end{array}$ & $\begin{array}{c}0.90 \mathrm{~L} / \mathrm{min} \\
\text { (constant) }\end{array}$ \\
\hline Arterial blood pressure (SD) & $\begin{array}{c}82.7 \mathrm{~mm} / \mathrm{Hg} \\
(\mathrm{SD} \mathrm{0.6)}\end{array}$ & $\begin{array}{c}80.7 \mathrm{~mm} / \mathrm{Hg} \\
(\mathrm{SD} 1.2)\end{array}$ & $\begin{array}{c}86.7 \mathrm{~mm} / \mathrm{Hg} \\
(\mathrm{SD} 1.2)\end{array}$ & $\begin{array}{c}88.7 \mathrm{~mm} / \mathrm{Hg} \\
(\mathrm{SD} 1.5)\end{array}$ & $\begin{array}{c}94 \mathrm{~mm} / \mathrm{Hg} \\
(\mathrm{SD} 2.7)\end{array}$ \\
\hline
\end{tabular}

SD: standard deviation.

was assessed by measuring the remaining luminal area on cross-sections. Mean diameter and area of the induced stenosis were compared to the FFR measurement to provide information on the severity of luminal narrowing. For myocardial evaluation, CT perfusion datasets were reconstructed in short-axis images with $3.0 \mathrm{~mm}$ slice thickness, $1.5 \mathrm{~mm}$ increment, and B23f (filtered back) reconstruction kernel for quantitative myocardial assessment, including an iodine beam hardening correction algorithm. Perfusion datasets were analysed using commercially available software, volume perfusion CT (VPCT) myocardium (VA41A, Siemens Healthcare, Forchheim, Germany). The AHA segmental model was used to label the segments of the heart [14]. The flow territory of the $\mathrm{Cx}$ was determined based on the total occlusion scan. Segments in the Cx territory were defined as Cx segments, others as non-Cx segments. Perfusion parameters for $\mathrm{Cx}$ myocardial segments and non-Cx segments were calculated. The FOV included the short-axis view of the heart. The inflow tube of the perfusate to the heart was looped through the FOV to allow calculation of an input function as reference for the perfusion of individual myocardial segments. The VPCT software uses the input function and the enhancement in the segments of the heart to calculate myocardial blood flow and blood volume in $\mathrm{mL} / 100 \mathrm{~mL} / \mathrm{min}$ and $\mathrm{mL} / 100 \mathrm{~mL}$, respectively, $100 \mathrm{~mL}$ being a measure for the volume of the myocardial tissue.

2.5. Statistical Analysis. Data management and statistical analysis were performed using Excel and SPSS 19 (IBM Corp, Armonk, NY). Independent variance tests were carried out to analyse whether the $\mathrm{Cx}$ and non-Cx segment groups showed normal distribution of perfusion measurements. Thereafter, the Mann-Whitney $U$ test for equality was performed. Median values of myocardial blood flow and volume were compared between segments with normal perfusion and segments with induced stenosis at different stenosis grades.

\section{Results}

The pacemaker-induced heart rhythm was stable at approximately 70 beats per minute (supplementary movie). Arterial blood flow and blood pressure were kept constant as much as possible at $1 \mathrm{~L} / \mathrm{min}$ and $80 \mathrm{~mm} / \mathrm{Hg}$, respectively. Blood flow was $1.0 \mathrm{~L} / \mathrm{min}$ at FFR values of $1.0,0.7$, and 0.5 and slightly lower, $0.9 \mathrm{~L} / \mathrm{min}$, at FFRs of 0.3 and $0.1 / 0.0$ (Table 1). Mean arterial pressure gradually increased from 80 to $95 \mathrm{~mm} / \mathrm{Hg}$ over the course of the experiment. Stenosis induction with FFR pressures of 0.3 and 0.0 caused the arterial pressure to increase with $10 \mathrm{~mm} / \mathrm{Hg}$ and blood flow to decrease with
TABLE 2: Diameter of stenosis divided by the artery diameter before stenosis, and the area stenosis divided by the area before stenosis, for the FFR-based stenosis grades.

\begin{tabular}{lccc}
\hline & FFR 0.70 & FFR 0.50 & FFR 0.30 \\
\hline Mean diameter on CTA & $74 \%$ & $47 \%$ & $31 \%$ \\
Area stenosis on CTA & $49 \%$ & $22 \%$ & $12 \%$ \\
\hline
\end{tabular}

$0.1 \mathrm{~L} / \mathrm{min}$. The FFR measurements at each stenosis grade were stable. Occasionally, the pressure of the inflatable cuff lowered, recognisable as a rise in the FFR value. Then, the cuff was inflated again to maintain FFR within the 0.05 boundary from the goal stenosis grade. CTA measurements of the stenosis diameter and stenosis area are shown in Table 2. A 0.70 FFRbased stenosis corresponded with a 50 percent area stenosis on CTA.

Image quality of the coronary CTA scans was high, with $\mathrm{HU}$ values of over 325 in the coronary arteries (Figure 2). In the dynamic scans, the heart could be imaged in total (Figure 3). Based on the total occlusion scan, the flow territory of the Cx was found to be limited to segment 5 (Figures 2 and 4). The relatively limited extent of ischemia was due to a small size Cx artery in this porcine heart.

At every stenosis grade, three scans with one stenotic Cx segment and 15 normally perfused segments were analysed, a total of 48 segments per stenosis grade. In Table 3 the median values of myocardial blood flow and blood volume are shown for $\mathrm{Cx}$ - and non-Cx-perfused segments. Myocardial blood flow and volume for the $\mathrm{Cx}$ segment were lower at stenosis grades with FFR of $\leq 0.50$ compared to non-Cx segments.

\section{Discussion}

This study shows the feasibility of myocardial perfusion analysis in a Langendorff pig heart model in a CT environment. This experimental setup enables detailed and systematic study of myocardial perfusion under standardized conditions and at differing degrees of blood flow, allowing for qualitative and quantitative evaluation of myocardial perfusion. Preliminary results suggest that newly developed CT perfusion imaging techniques can be validated with this model under controlled conditions, with hemodynamic settings relatively similar to the in vivo, clinical situation.

The Langendorff model is a retrograde perfusion model established in 1895 [11]. In 2007, Skrzypiec-Spring et al. reviewed the use of this model throughout a century of existence and concluded that it can be used to study ischaemia, 


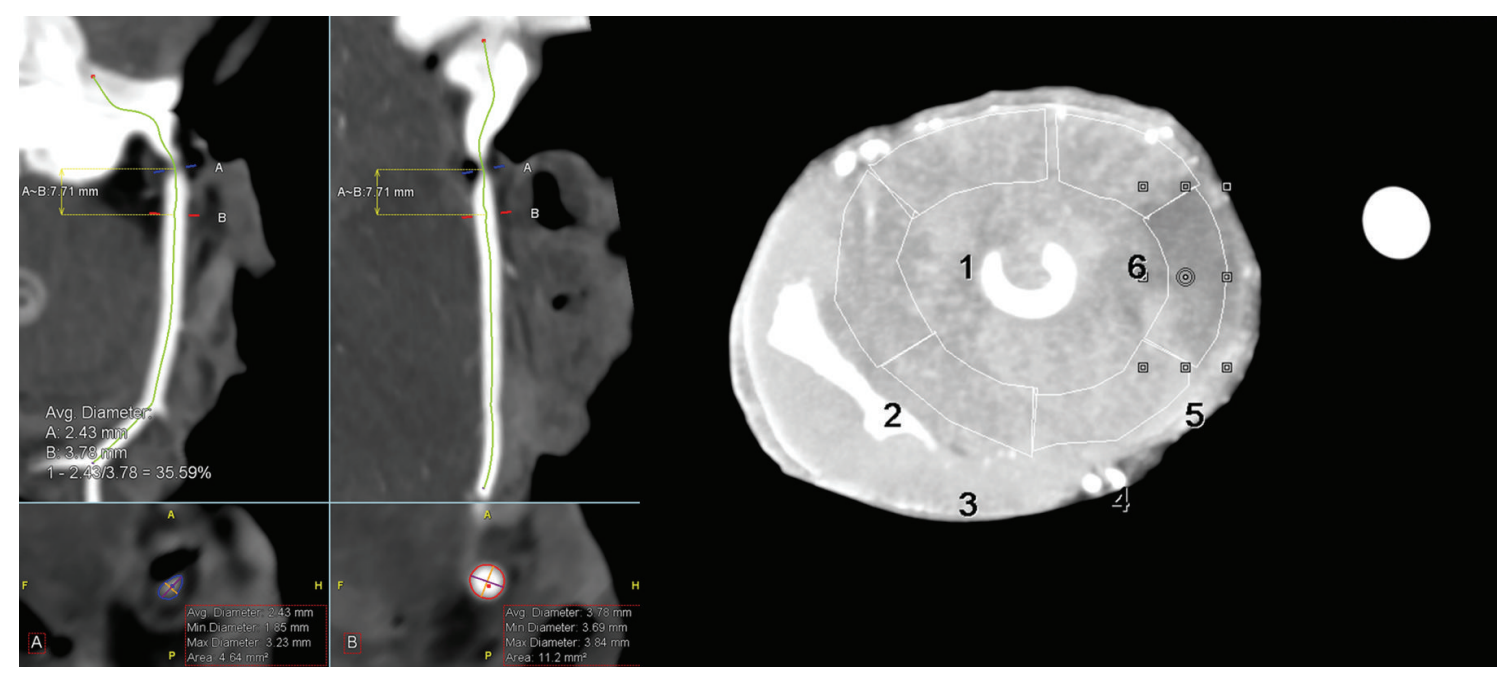

(a)

(b)

FIgURE 2: Example of images of the Cx artery with an FFR of 0.30 . (a) The computed tomography angiography (CTA) image in curved multiplanar reformat shows the luminal narrowing. (b) The resulting perfusion defect in segment 5 as visible on maximum intensity projection (MIP) image.

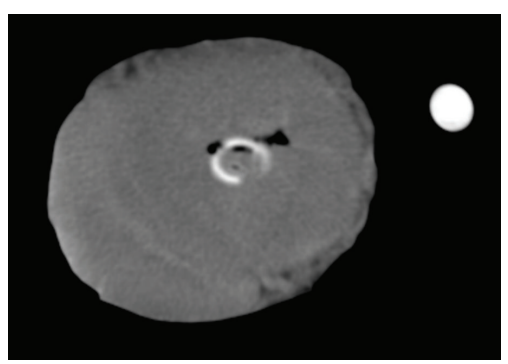

(a)

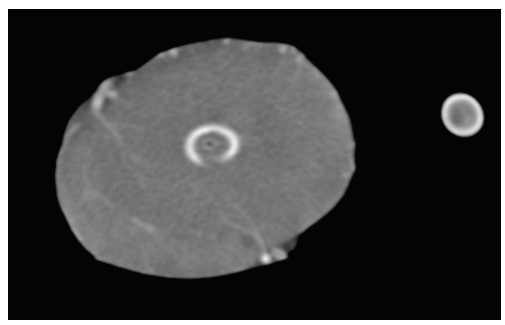

(d)

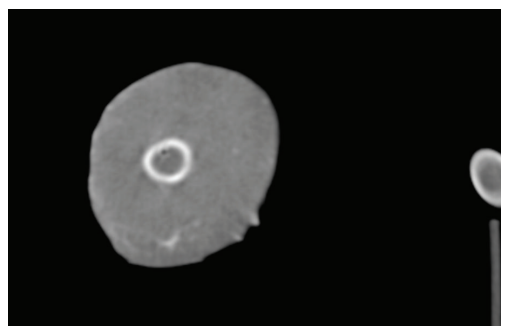

(g)

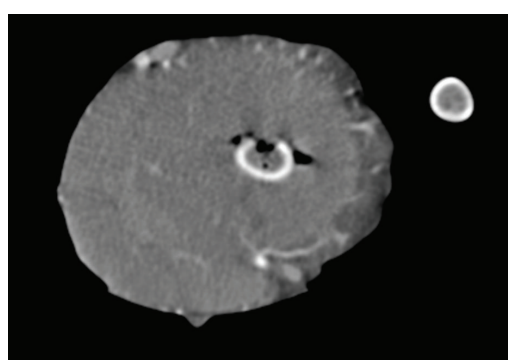

(b)

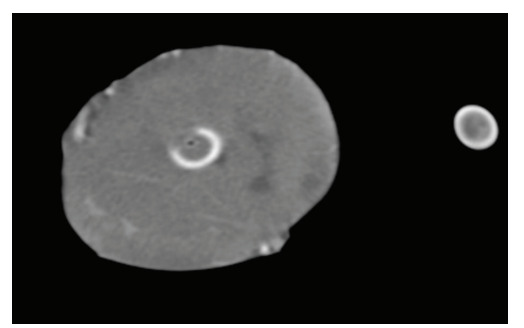

(e)

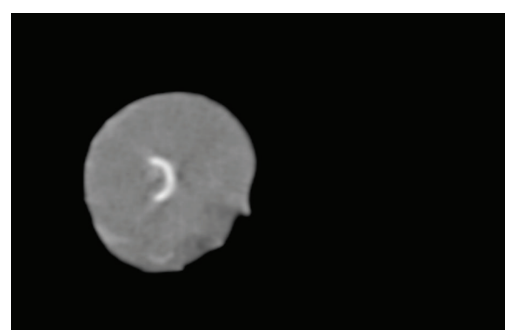

(h)

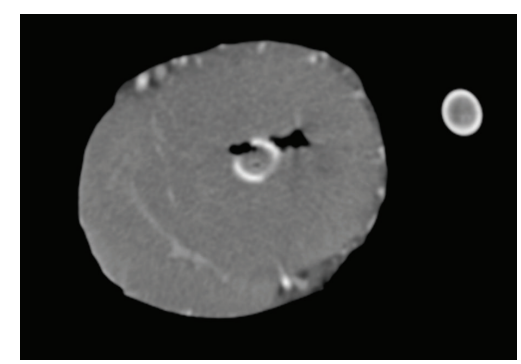

(c)

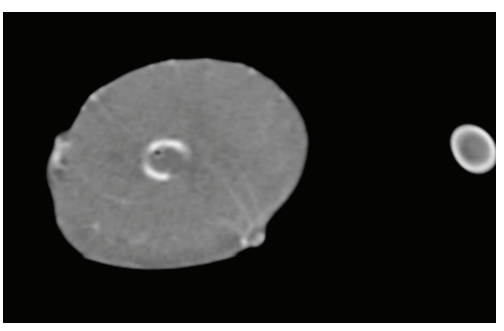

(f)

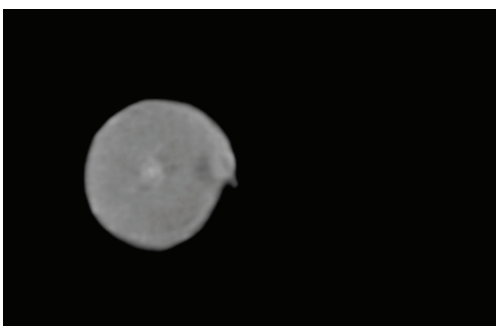

(i)

FIGURE 3: Image (a) shows the inflow of CM bolus through the inflow tube. Images (b)-(i) show the contrast inflow in the myocardium at short-axis cross-sections from basal to apical. 
TABLE 3: Median blood flow (L/min) in normal and defected segments for multiple stenosis grades.

\begin{tabular}{|c|c|c|c|c|c|}
\hline & No stenosis & FFR 0.70 & FFR 0.50 & FFR 0.30 & Total occlusion \\
\hline $\begin{array}{l}\text { Calculated normal } \\
\text { segments (min-max) }\end{array}$ & $\begin{array}{c}151 \mathrm{~mL} / 100 \mathrm{~mL} / \mathrm{min} \\
(113-205)\end{array}$ & $\begin{array}{c}173 \mathrm{~mL} / 100 \mathrm{~mL} / \mathrm{min} \\
(122-260)\end{array}$ & $\begin{array}{c}162 \mathrm{~mL} / 100 \mathrm{~mL} / \mathrm{min} \\
(133-247)\end{array}$ & $\begin{array}{c}124 \mathrm{~mL} / 100 \mathrm{~mL} / \mathrm{min} \\
(70-207)\end{array}$ & $\begin{array}{c}108 \mathrm{~mL} / 100 \mathrm{~mL} / \mathrm{min} \\
(43-167)\end{array}$ \\
\hline $\begin{array}{l}\text { Calculated defect } \\
\text { segments (min-max) }\end{array}$ & $\begin{array}{c}133 \mathrm{~mL} / 100 \mathrm{~mL} / \mathrm{min} \\
(127-139)\end{array}$ & $\begin{array}{c}157 \mathrm{~mL} / 100 \mathrm{~mL} / \mathrm{min} \\
(129-183)\end{array}$ & $\begin{array}{c}121 \mathrm{~mL} / 100 \mathrm{~mL} / \mathrm{min} \\
(96-125)\end{array}$ & $\begin{array}{c}74 \mathrm{~mL} / 100 \mathrm{~mL} / \mathrm{min} \\
(66-78)\end{array}$ & $\begin{array}{c}34 \mathrm{~mL} / 100 \mathrm{~mL} / \mathrm{min} \\
(34-39)\end{array}$ \\
\hline
\end{tabular}

Minimum and maximum blood flow for each group are shown in brackets.

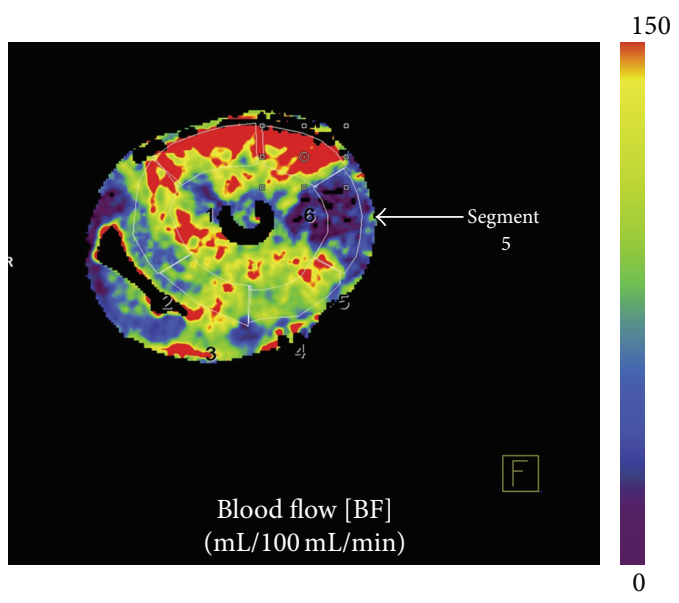

Figure 4: Blood flow map of the porcine heart at complete Cx occlusion: red color meaning higher/normal flow and blue indicating reduced flow.

stunning, hibernation, and arrhythmias and for drug testing [10]. In several studies the Langendorff model has been used to investigate, for instance, cardiac physiology and donor heart preservation methods $[15,16]$. In our study, arterial blood flow and pressure were stable and controllable during most of our experiment. Part of the controllable circumstances included the confirmation of the stenosis degree by FFR assessment. The decrease in blood flow and increase in blood pressure during the experiment can be explained by the increase in peripheral resistance due to the induced stenosis. Because the heart was explanted, it was already in stress state and no compensatory mechanisms were present [9]. Thus, induction of a stenosis directly influenced coronary blood flow and pressure. A stenosis with a 0.7 pressure drop, however, did not influence blood flow and blood pressure in this single experiment. We hypothesize that the lumen area may still be too large for peripheral resistance to increase, and, therefore, blood flow and arterial pressure will not be influenced.

Schuster et al. showed potential for the Langendorff model for perfusion analysis in MRI environment [17]. They showed consistent blood flow and pressure over time for hearts without stenosis. However, they did not evaluate the effect of increasing coronary stenosis on model stability (RCA in one heart was occluded but only to compare first pass perfusion area to infarct size).

Small clinical studies have shown that adenosine stress dynamic perfusion CT testing can detect hemodynamically significant stenosis [3, 4, 18-20]. Quantitative evaluation of myocardial perfusion may have incremental value to detect flow-limiting stenosis compared to visual analysis. Prior to clinical implementation, the relationship between CTderived perfusion parameters across the range of coronary stenosis needs validation against reference standards. Ethically, it is difficult to justify comparison of two modalities with associated radiation dose in patients. Also, there is little control over hemodynamic parameters in a human model especially regarding degree of stenosis. Conversely, a phantom model only allows limited conclusions with regard to the in vivo situation. An animal model mimicking human cardiac circulation may be of great help. Pigs have been researched extensively in cardiac imaging, since the porcine model provides excellent comparison to the human heart in size and physiology [21-24]. The porcine heart is especially well suited for myocardial perfusion imaging studies, because it cannot develop collateral flow. Thus, there is a direct relationship between stenosis grade and the decrease in downstream perfusion.

In in vivo porcine experiments CT allowed quantification of coronary flow [5, 22-25]. The advantage of in vivo experiments is that the physiology and cardiac function is intact providing cardiac features comparable to human studies. However, in vivo experiments are often more complex than single-heart experiments, because whole pigs cannot as easily and directly be manipulated as explanted hearts. Physiologically, the explanted heart is not as complex as the heart in living animals, offering more control and possibly better reproducibility. An extension of the present experiment could lie in imaging the porcine heart with different modalities to directly compare perfusion techniques at different stenosis grades.

Summarizing, our model has several benefits compared to other experimental models. A major advantage of the Langendorff model is the control of myocardial blood flow. A stenosis can be directly induced, and its severity can easily be altered. Because the heart is disconnected from the body, it is free of neural/hormonal influences. The heart is already in stress state, because of the prior explantation. Thus, the coronary arteries are at maximum dilation, mimicking the situation of adenosine stress perfusion imaging. Furthermore, after otherwise fatal events such as arrhythmias and infarction-induced cardiac arrest, introducing a pacemaker or reinstating sinus rhythm with defibrillations of 10-30 J can prolong the experiment. Compared to scanning and subsequent sacrificing of living pigs, a model using slaughterhouse pigs provides an alternative where no additional animals are sacrificed. 
Our model also has some disadvantages. Due to the fact that physiological processes were no longer intact, the model is less comparable to the clinical situation. The setup platform and fixation as well as contrast protocol and left ventricular pressure are not like in vivo situations. The setup was mostly made of plastic, because metal would induce large artifacts. Furthermore, the heart experienced an ischaemic period directly after removal, which may alter its condition and influence perfusion. During the experiment, some difficulties in ECG-triggering were experienced. This could be due to the usage of the pacemaker. The signal transduction of the pacemaker spike within the heart can differ between beats, possibly resulting in phase irregularities. Even so, signal transduction during the experiment was generally good, with good image quality and limited motion artifacts. Pacemakerinduced rhythm is generally stable; however premature ventricular contractions may occur. Another possible bias is the fixed pacemaker-induced heart rate in this heart. Previous studies in humans have shown an increase in heart rate when stressed using adenosine $[18,19]$. This increase is not simulated in this particular pig heart experiment. Therefore, our Langendorff experiment could be less prone to heart rate dependent artefacts compared to human studies when heart rates are higher. The stenosis induced in the $\mathrm{Cx}$ artery did not cause a large perfusion defect, because of a rudimentary $\mathrm{Cx}$ artery. There is a natural distribution in the size and perfusion areas of the coronary arteries in pigs, comparable to humans. We intend to repeat this experiment in a number of pig hearts to gain more information on variability of the model. Compared to an entire pig, the current model is relatively expensive. A last limitation is that the study was performed in one pig heart, only. The current study was designed to purely show the feasibility of the model. The model should be repeated in a larger number of explanted hearts to confirm stability of the setup and to optimize the experimental protocol.

\section{Conclusion}

This study demonstrates the feasibility of ex vivo myocardial perfusion imaging and quantification in a CT environment at different grades of coronary stenosis. The Langendorff model provides control over physiological parameters such as blood flow and stenosis grade. The described model shows promise for validation of CT perfusion imaging techniques under controlled circumstances.
Abbreviations
CAD: Coronary artery disease
CM: Contrast media
CT: Computed tomography
CTA: Computed tomography angiography
CTP: Computed tomography perfusion
Cx: Circumflex artery
FOV: Field of view
FFR: Fractional flow reserve
MRI: Magnetic resonance imaging
DSCT: Dual source computed tomography
PET: Positron emission tomography

RCA: Right coronary artery

VPCT: Volume perfusion CT.

\section{Conflict of Interests}

Ulrike Haberland and Ernst Klotz are employees of the CT Division of Siemens Healthcare Sector (Forchheim, Germany) which manufactured the CT equipment used in this study. Sjoerd van Tuijl and Marco Stijnen are employees of LifeTec Group (Eindhoven, Netherlands) which provided the Langendorff porcine heart setup as well as the porcine heart itself.

\section{Acknowledgment}

Rozemarijn Vliegenthart is supported by a grant from the Netherlands Organisation for Scientific Research.

\section{References}

[1] J. D. Schuijf, W. Wijns, J. W. Jukema et al., "A comparative regional analysis of coronary atherosclerosis and calcium score on multislice CT versus myocardial perfusion on SPECT,' Journal of Nuclear Medicine, vol. 47, no. 11, pp. 1749-1755, 2006.

[2] A. Rossi, D. Merkus, E. Klotz, N. Mollet, P. J. de Feyter, and G. P. Krestin, "Stress myocardial perfusion: imaging with multidetector CT," Radiology, vol. 270, no. 1, pp. 25-46, 2014.

[3] Y. Wang, L. Qin, X. Shi et al., "Adenosine-stress dynamic myocardial perfusion imaging with second-generation dualsource CT: comparison with conventional catheter coronary angiography and SPECT nuclear myocardial perfusion imaging," American Journal of Roentgenology, vol. 198, no. 3, pp. 521529,2012

[4] F. Bamberg, A. Becker, F. Schwarz et al., "Detection of hemodynamically significant coronary artery stenosis: incremental diagnostic value of dynamic CT-based myocardial perfusion imaging," Radiology, vol. 260, no. 3, pp. 689-698, 2011.

[5] A. H. Mahnken, E. Klotz, H. Pietsch et al., "Quantitative whole heart stress perfusion CT imaging as noninvasive assessment of hemodynamics in coronary artery stenosis: preliminary animal experience," Investigative Radiology, vol. 45, no. 6, pp. 298-305, 2010.

[6] A. M. Huber, V. Leber, B. M. Gramer et al., "Myocardium: dynamic versus single-shot CT perfusion imaging," Radiology, vol. 269, no. 2, pp. 378-386, 2013.

[7] P. A. Kaufmann and P. G. Camici, "Myocardial blood flow measurement by PET: technical aspects and clinical applications," Journal of Nuclear Medicine, vol. 46, no. 1, pp. 75-88, 2005.

[8] G. Morton, A. Chiribiri, M. Ishida et al., "Quantification of absolute myocardial perfusion in patients with coronary artery disease: comparison between cardiovascular magnetic resonance and positron emission tomography," Journal of the American College of Cardiology, vol. 60, no. 16, pp. 1546-1555, 2012.

[9] S. Schampaert, M. van 't Veer, M. C. M. Rutten et al., "Autoregulation of coronary blood flow in the isolated beating pig heart," Artificial Organs, vol. 37, no. 8, pp. 724-730, 2013.

[10] M. Skrzypiec-Spring, B. Grotthus, A. Szelag, and R. Schulz, "Isolated heart perfusion according to Langendorff-still viable in the new millennium," Journal of Pharmacological and Toxicological Methods, vol. 55, no. 2, pp. 113-126, 2007. 
[11] O. Langendorff, "Untersuchungen am überlebenden Säugethierherzen," Pflüger, Archiv für die Gesammte Physiologie des Menschen und der Thiere, vol. 61, no. 6, pp. 291-332, 1895.

[12] D. Modersohn, S. Eddicks, C. Grosse-Siestrup, I. Ast, S. Holinski, and W. Konertz, "Isolated hemoperfused heart model of slaughterhouse pigs," International Journal of Artificial Organs, vol. 24, no. 4, pp. 215-221, 2001.

[13] F. Bamberg, E. Klotz, T. Flohr et al., "Dynamic myocardial stress perfusion imaging using fast dual-source CT with alternating table positions: initial experience," European Radiology, vol. 20, no. 5, pp. 1168-1173, 2010.

[14] M. D. Cerqueira, N. J. Weissman, V. Dilsizian et al., "Standardized myocardial segmentation and nomenclature for tomographic imaging of the heart. A statement for healthcare professionals from the Cardiac Imaging Committee of the Council on Clinical Cardiology of the American Heart Association," Circulation, vol. 105, no. 4, pp. 539-542, 2002.

[15] W. F. Fearon, L. B. Balsam, H. M. O. Farouque et al., "Novel index for invasively assessing the coronary microcirculation," Circulation, vol. 107, no. 25, pp. 3129-3132, 2003.

[16] H. Aupperle, J. Garbade, C. Ullmann et al., "Comparing the ultrastructural effects of two different cardiac preparationand perfusion-techniques in a porcine model of extracorporal long-term preservation," European Journal of Cardio-Thoracic Surgery, vol. 31, no. 2, pp. 214-221, 2007.

[17] A. Schuster, I. Grünwald, A. Chiribiri et al., "An isolated perfused pig heart model for the development, validation and translation of novel cardiovascular magnetic resonance techniques," Journal of Cardiovascular Magnetic Resonance, vol. 12, article 53, 2010.

[18] G. Bastarrika, L. Ramos-Duran, M. A. Rosenblum, D. K. Kang, G. W. Rowe, and U. J. Schoepf, "Adenosine-stress dynamic myocardial CT perfusion imaging: initial clinical experience," Investigative Radiology, vol. 45, no. 6, pp. 306-313, 2010.

[19] K. T. Ho, K. C. Chua, E. Klotz, and C. Panknin, "Stress and rest dynamic myocardial perfusion imaging by evaluation of complete time-attenuation curves with dual-source CT," JACC: Cardiovascular Imaging, vol. 3, no. 8, pp. 811-820, 2010.

[20] M. Weininger, U. J. Schoepf, A. Ramachandra et al., "Adenosinestress dynamic real-time myocardial perfusion CT and adenosine-stress first-pass dual-energy myocardial perfusion CT for the assessment of acute chest pain: initial results," European Journal of Radiology, vol. 81, no. 12, pp. 3703-3710, 2012.

[21] D. J. Hearse and F. J. Sutherland, "Experimental models for the study of cardiovascular function and disease," Pharmacological Research, vol. 41, no. 6, pp. 597-603, 2000.

[22] A. Rossi, A. Uitterdijk, M. Dijkshoorn et al., "Quantification of myocardial blood flow by adenosine-stress CT perfusion imaging in pigs during various degrees of stenosis correlates well with coronary artery blood flow and fractional flow reserve," European Heart Journal Cardiovascular Imaging, vol. 14, no. 4, pp. 331-338, 2013.

[23] F. Schwarz, R. Hinkel, E. Baloch et al., "Myocardial CT perfusion imaging in a large animal model: comparison of dynamic versus single-phase acquisitions," JACC: Cardiovascular Imaging, vol. 6, no. 12, pp. 1229-1238, 2013.

[24] A. So, J. Hsieh, J. Y. Li, J. Hadway, H. F. Kong, and T. Y. Lee, "Quantitative myocardial perfusion measurement using CT Perfusion: a validation study in a porcine model of reperfused acute myocardial infarction," International Journal of Cardiovascular Imaging, vol. 28, no. 5, pp. 1237-1248, 2012.
[25] F. Bamberg, R. Hinkel, F. Schwarz et al., "Accuracy of dynamic computed tomography adenosine stress myocardial perfusion imaging in estimating myocardial blood flow at various degrees of coronary artery stenosis using a porcine animal model," Investigative Radiology, vol. 47, no. 1, pp. 71-77, 2012. 


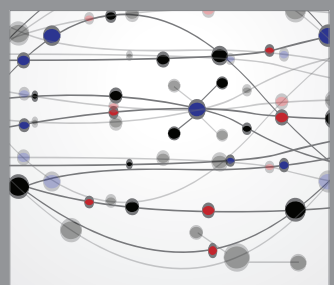

The Scientific World Journal
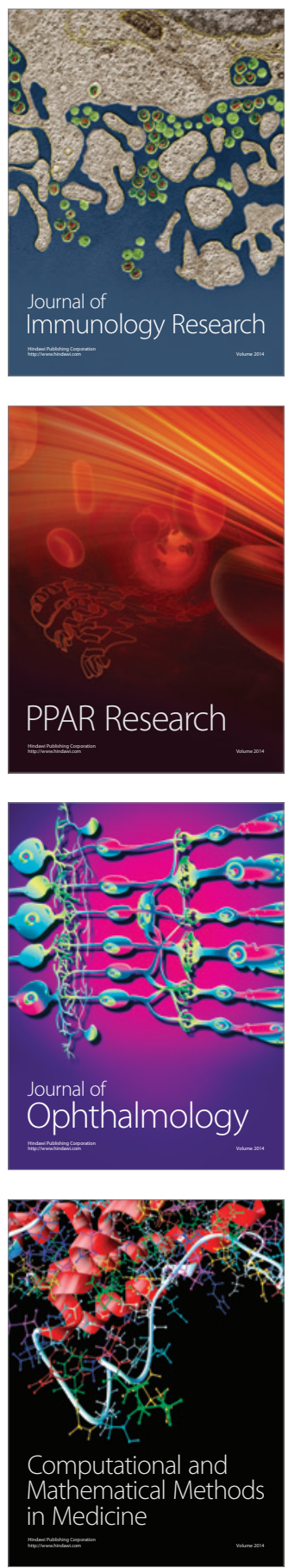

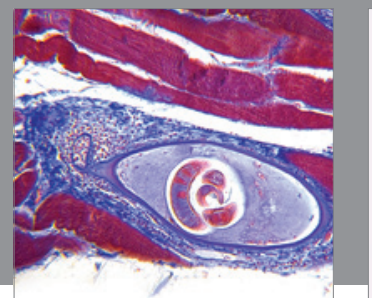

Gastroenterology

Research and Practice
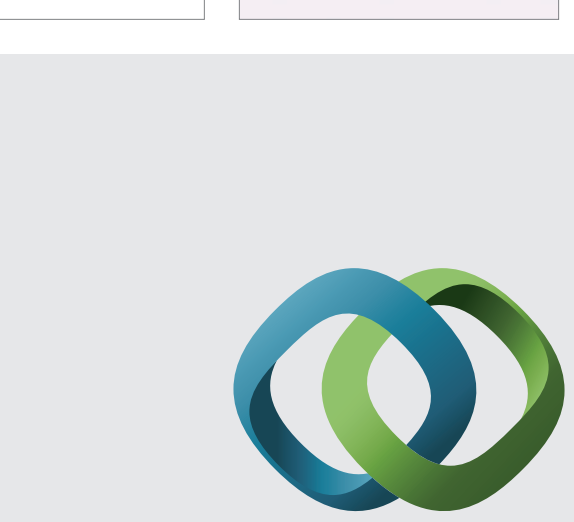

\section{Hindawi}

Submit your manuscripts at

http://www.hindawi.com
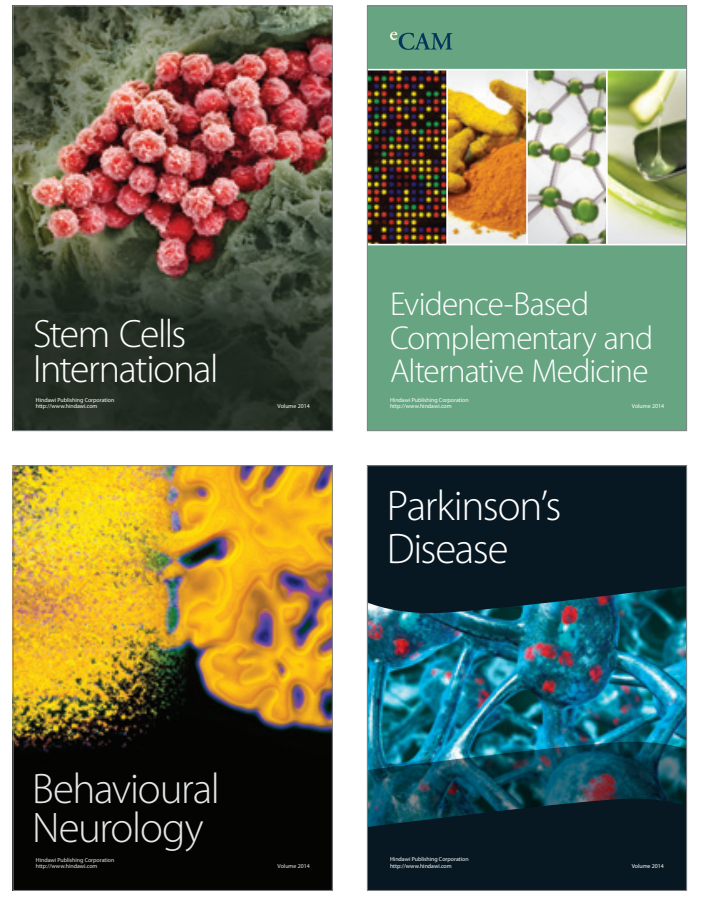
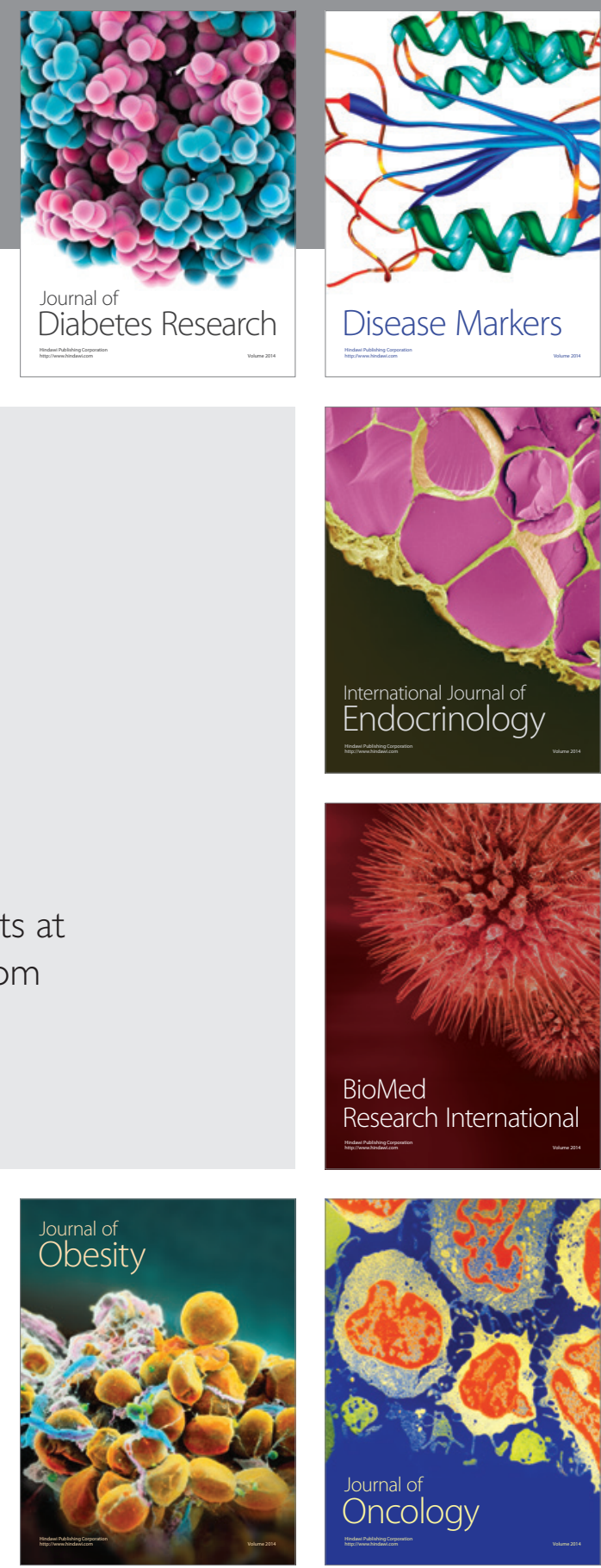

Disease Markers
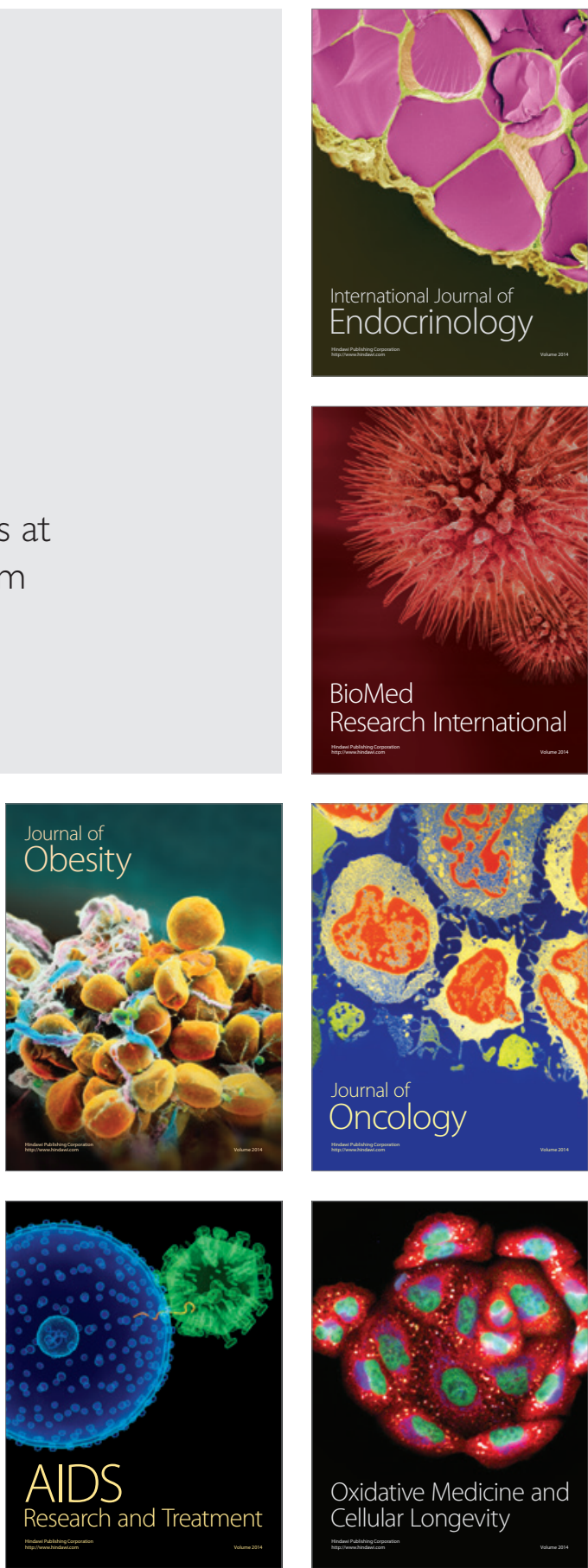